\title{
Wanting a Life in Decency!-A Qualitative Study from Experienced Electric Wheelchairs Users' perspective
}

\author{
Lisbeth Kristiansen ${ }^{1,2}$ \\ ${ }^{1}$ Department of Nursing Sciences, Faculty of Human Sciences, Mid Sweden University, Sundsvall, Sweden \\ ${ }^{2}$ Department of Health Care, Faculty of Sciences, University of Gävle, Gävle, Sweden \\ Email: Lisbeth.kristiansen@miun.se, Lisbeth.porskrog.kristiansen@hig.se
}

How to cite this paper: Kristiansen, L. (2018) Wanting a Life in Decency!-A Qualitative Study from Experienced Electric Wheelchairs Users' perspective. Open Journal of Nursing, 8, 419-433. https://doi.org/10.4236/ojn.2018.87033

Received: June 4, 2018

Accepted: July 13, 2018

Published: July 16, 2018

Copyright $\odot 2018$ by author and Scientific Research Publishing Inc. This work is licensed under the Creative Commons Attribution-NonCommercial International License (CC BY-NC 4.0). http://creativecommons.org/licenses/by-nc/4.0/ cc) (i) (8) Open Access

\begin{abstract}
Background: The functionality and the safety of the electric wheelchairs were essential for users' everyday life. Some evidence indicated that the wheelchair Per Se highly influenced users' occupational life, their personal identity and social life; further, the wheelchair became an extension of the body and more than a technical device. Besides, there was still both environmental and self-efficacy or/and mental health factors obstacles for full social participation. Even so, there was to some extent stigma related to being a wheelchair user. There was a need to reflect users' perspective on being depended on electric wheelchair. The aim, accordingly, was to describe and to get a deeper insight into electric wheelchairs users' perspective and experiences of utilizing this device; a qualitative design with an inductive approach was used. Method: Qualitative latent and interpretative content analysis [1] [2] was used after repeated face-to-face semi-structured interviews with three experienced Swedish electric wheelchair users during the autumn 2017. Findings: The findings showed a high degree of dependability of the assistants that supported the users, and of the quality of that working relationship. The findings were formulated, abstracted and interpreted in several steps. It showed one theme of meaning: "Living in a space shifting between potential violation of or respect for human dignity". Conclusion: The study showed that electric wheelchair users were relatively content with their lives as well as with their devices in turns of mobility and accessibility, but the meaning of their narrations showed a life at constant risk of having the respect of human rights and human respect violated. Besides, the importance of having access to good and high quality devices, good staffing, and environmental support, all in concordance with human rights, the clinical and practical implications of this study nar-
\end{abstract}


rows down to a question of encountering the other person as a whole and worthy individual.

\section{Keywords}

Electric Wheelchair, Qualitative Latent Analysis, Users’ Perspective

\section{Introduction}

\subsection{A matter of Human Rights}

This paper focuses on people in electric wheelchair and their perspective on how that is experienced. According to the "Convention on the Rights of Persons with Disabilities", all United National member states must take measures that ensure personal mobility with the greatest possible independence for persons with disabilities [3]. This, among other things, included: (b) Facilitating access by persons with disabilities to quality mobility aids, devices, assistive technologies and forms of live assistance and intermediaries, including by making them available at affordable cost [3 Article 20, b]. The convention was ratified 2009 by Sweden. All Swedish citizens had a birthright to benefit from the public supported subventions regarding wheelchair devices, which occasionally included electric wheelchairs. Further, the World Health Organization's International Classification of Functioning, Disability, and Health (ICF) model of disability integrates the medical, social, and functional dimensions and provides a positive, enablement-focused, rather than disability-oriented, framework. It views disability as restriction in participation in life activities and as an interactive construct [4]. The ICF assesses domains such as body function and structure, activity and participation, and personal and environmental factors, such as access to transportation [5]. In the ICF, functional limitations of impairment become disabling in the context of broader physical, social, and attitudinal factors [6]. There is an emphasis on using measures of constructs mapped by the ICF and recommend linking the ICF-postulated assessment model to individual and social interventions [7].

\subsection{Perspective of the End User}

The ability for people with mobility impairments to live in their homes and communities with maximal independence often hinges, in part, on their ability to transfer or to be transferred by an assistant. The lived experiences of nine children and young people who were users of an Electric Powered Indoor/Outdoor wheelchair (EPIOC), revealed that they worked to achieve an adequate fit between self, the EPIOC and the environment [8]. Positive consequences of using an EPIOC were that it increased the informants' participation and positive feelings. However, an inadequate fit led to negative experiences in- 
cluding reduced participation, fear and anxiety. The changing interface between self, EPIOC and environment appeared to be important in shaping these experiences. These ambiguous findings is important to keep in mind, as children and young people are active agents in shaping their experiences, the need for ongoing support from services especially around stages of transition. It also contributed to the development of the social model of disability from a child's perspective [8]. While, other adult users of electric wheelchairs experienced that the device helped them in their everyday life [9]. They spook about the importance of personal help and the appropriate design of their home. The participants' lives were considerably influenced by their aids, either by increasing or by limiting their occupational possibilities in everyday life at micro- or meso-levels. This also influenced their social life and identity at a macro level. Taken together, the participants expressed a wish to be independent and equal individuals, and wanted to have the opportunity to follow their individual wishes at all levels regarding occupation and life course. In addition, they expressed having been able to adapt to the situation in different ways and to get the best out of life with a functional impairment. Four central themes in the participants' experiences is about their everyday life as users of an electric wheelchair. The dominating problem, which limited the users' occupation in relation to the wheelchair, was the electric wheelchair's battery. All nine participants mentioned that they had problems with the wheelchair's battery, in part because the battery was big and heavy, thereby influencing the weight of the wheelchair, and in part, because the capacity of the battery was too small. This meant that the wheelchair had to recharge for several hours every day, and that the users had to adapt this resulting in immobility during these hours every day. As the battery only had a limited capacity, it overall limited the participants' aspirations and plans for daily occupation in accordance with their wishes and values [9]. Along the same line, twelve experienced power users emphasized that electric wheelchair-driving difficulties were related to the accomplishment of activities of daily living, and the influence of environmental context [10]. Four key themes emerged: 1) difficulties accessing and using public buildings-facilities, 2) outdoor mobility, 3) problems in performing specific wheelchair mobility tasks/maneuvers and 4) barriers and circumstances that are temporary, unforeseen or specific to a particular context. Their contribution was an enhanced understanding of the driving difficulties that powered wheelchair users' experienced during daily activities including ecologically validated measurements for power wheelchair users, and identifying and refining the content of training programs specific to the use of power wheelchairs [10]. A recently published Swedish study showed that electric wheelchair users embodied their experiences of being a wheelchair user, and the wheelchair became much more than a device that helped them to mobility [11].

However, the electric wheelchair users were constantly confronted with stigma, because of the status of the disabled in society, which, sometimes isolated the users and negatively affected their self-concepts and identity [9], while, other 
adolescents only to a little extend displayed stigma towards wheelchair users [12].

\subsection{Challenges Related to Wheelchair Use}

There are several challenges related to using a wheel chair. Grindles et al. [13] have seen that between the years 1973 and 1987, 770 lethal wheelchair-related accidents were reported to the US Consumer Products Safety Commission. 8.1\% of these accidents were caused by falls during transfers [14]. Between the years 1986 and 1990, an estimated 36,000 wheelchair-related accidents in the US that resulted in a need to visit the emergency department, and $17 \%$ occurred due to falls during transfers [15]. In 2003, more than 100,000 wheelchair-related injuries were treated in US emergency departments, showing an upward trend in the number of injuries over time [16]. There are no such numbers available in the Swedish context to the author's knowledge.

\subsection{Social Inclusion and Community Participation}

The opposite of social inclusion is social isolation, which may defined as "a state in which the individual lacks sense of belonging socially, lacks engagement with others, has a minimum of social contacts and they are deficient in fulfilling relationship" Nicholson [17]. The European Union has on the other hand, defined social inclusion, as having the opportunities and resources to participate fully in economic, social and cultural life and to enjoy a standard of well-being that is considered normal in the society in which we live [18]. Social inclusion can increase mental health and reduce mental illness, help to promote recovery, and provide both mental and physical health gains. A Canadian study showed that wintertime is certain challenge for users of wheeled mobility devices (scooters, manual and power wheelchairs) and three firth approximate 80 uses reported that they needed additional help during that season [19]. This could lead to a limited access to the surrounding community and to a sense of loneliness/isolation, and fear/anxiety related to safety. There is a decrease in community participation in cold weather because of multiple environmental barriers. Clinicians, researchers, and policymakers can take a multidimensional approach to mitigate these barriers in order to enhance community participation for wheeled mobility devices users in winter [19].

In a review of literature based on thirty-five studies, Smith, Sakakibara, and Miller [20] concluded the social and community participation of wheelchair users was complex phenomenon. It was influenced by all of the body structures and functions, activity and participation, as well as and environmental and personal factors. Overall, the review showed that wheelchair factors and accessibility the most frequently reported factors associated with participation, while wheelchair skills, pain, finances and level of education were common modifiable factors. The authors pointed out the lack of efficacy studies in the relation to enhanced societal participation with interventions that should impact users' im- 
proved wheelchair confidence and/or lowered depression [20].

\subsection{Theoretical Framework}

This study had a social-ecological theory base, which assumed that a person's health is influenced by dynamic interactions between personal characteristics, including the genetic, behavioral patterns, mental state and the surrounding environment [21]. This is also in line with Bronfenbenner's theory [22] that adjacent micro-, meso-, exo-, macro-, and chrono-systems and the interplay between the systems can have direct or indirect influence on and influence on human health. The present study, tried to understand and take into account the health of these people as a whole, where mobility has an impact on their lives as a whole.

\subsection{Rational}

From a world-community perspective, the will of equal human rights for all people is evident. The functionality and the safety of the electric wheelchairs were essential for users' everyday life. Furthermore, there are some evidence showing that the wheelchair Per Se highly influenced users' occupational life, their personal identity and social life, that the wheelchair became an extension of the body and more than a technical device. Besides, there was still both environmental and self-efficacy or/and mental health factors obstacles for full social participation. Even so, there was to some extent stigma related to being a wheelchair user. There is a need to further reflect the users' perspectives on being depended on electric wheelchair. This study, therefore, seeks to contribute to filling the gap accordingly.

The aim of the study was to gain a deeper insight into electric wheelchairs users' perspective and experiences of utilizing this device.

\section{Method}

The study had a qualitative approach and a descriptive design with manifest and latent content analysis inspired by [1] [2] to gain insight into and understanding of the variation of how end users' of electrified wheelchairs experiences their lives and their devices. The author chose a qualitative approach as it matched the assumptions of the underlying naturalistic paradigm [23]. The naturalist paradigm ontologically assumed the existence of multiple and subjective realities; whereas it is epistemologically possible to acquire knowledge by attempting to understand people's life-world by interviewing them ([1] [24], pp. 13-14]).

\subsection{The Local Research Context}

The study was conducted in the Northern part of Sweden in a region with approximately 240,000 inhabitants divided in seven municipalities in the largest municipality with one hundred thousand inhabitants. The main occupations were within the areas of public health care, community service, education, tour- 
ism, smaller private entrepreneurs, and a few larger paper and chemical industries. Around 40 percent had university education. A large proportion is older and retired people.

\subsection{Sampling}

The sampling strategy is purposeful [23], containing two distinct phase; one there the head of department for the municipality's, the public assistant service, identified approximately five electric wheelchair end users over the age of 18 years. The head of department contacted these though ordinary post with information about the project's goals, and with contact information on the responsible researcher to get more information, if interested in this phase. This sampling procedure was chosen to secure that the end users were blinded from the research team.

The second phase started when users on their own initiative actively contacted the researcher by e-mail or mail to learn more about the project. The study population consisted of three end users. During this second phase, they were given accurate information on their participation, and this included giving information on what informed consent meant. Firstly, the research team decided on participation feasibility according to the following criteria.

\section{Inclusion and Exclusion Criteria for Study Participation}

Inclusion criteria were adult people with more than two years of experience of being an electric wheel chair user, and being able to communicate in Swedish. Exclusion criteria were an ongoing acute mental illness.

\subsection{Participants}

The participants in this study were three electric wheelchair users. In order to protect the identity of the participants, only few interviewee details are provided. All participants were older than 18 years, and ethnic Swedes. Some of them used the electric wheelchair as the person was injured in an accident. One had been a user since birth due to illness, and the last end user had residual symptoms after an illness. Two of them were employed and one retried. All of wheelchair users had three to four different assistants that were employed and financed by the municipality. Ever thought, the single user formally hired and fired the assistants. The assistants typically came the user's private home every day. Two of the participants had constant supervision 24 hour a day, and one had supervision several times a day and in the night. The assistants were all females with working experience of this work between one and teen years.

\subsection{Data Collection}

Individual semi-structured qualitative face-to-face interviews with three users were conducted twice in their homes and/or at the university as suggested by the participants. The total interview duration was around six hours. The participants were asked to narrate freely about their lives, by posing a wider and open ques- 
tion: "Can you, please, tell me about your experience it to be an electric wheelchair user". Further, questions like "How is your life", and "What are the benefits and challenges according to you" were asked. Clarifying and exemplifying questions were posed to deepen the understanding.

\subsection{Data Analysis}

The transcribed interviews were subsequently analyzed by qualitative content analysis [1] [2] [25]. The analysis started with several re-reading of the approximately 40 double spaced pages, focusing on what participants actually expressed during the interviews. Meaning units were identified, which were based on text that dealt with the same content. These meaning units were when condensed while retaining their essential meaning. A coding procedure then followed; where all condensed meaning units got a label, just stating the content of it. The next step was coding of the condensed units. Afterwards, I sorted the codes into sub-categories. Further, based on interpretation and abstraction of how the experiences appeared, categories were created, while keeping the study as a whole in mind. By asking questions to the interview text on: "What are participants trying to tell me?" descriptive themes were created, and by asking, "What was the meaning of the electric wheelchair users' stories", the author created a theme of meaning [2]. This corresponded to a latent and interpretive content analysis [2].

\subsection{Ethical Consideration}

This research was conducted in the recognition of the Madrid Declaration on Ethical Standards for Psychiatric Practice [26]. It is stated that ethical standards should also be applied in the selection of population groups. In all types of research including epidemiological and sociological studies and in collaborative research involving other disciplines or several investigating centers, for instance, the clients, end users were accepted as a partner by right in a relationship based on mutual trust and respect. Further, ethical permission is provided by regional committee of Ethical permission, in Umeå, Sweden, reference number 2017/283-31.

\section{Findings}

The findings showed that the users in general were satisfied with their lives and their wheelchairs' functionality including with the mobility of the electric wheelchair gave them. They shared their experiences on feeling relatively free and mobile. Further, they narrated stories about how some assistants did not pay adequately respect to them. The findings are created and constructed through abstractions and interpretation of what the participants shared in the repeated interviews. One theme of meaning; "Living in a space between potential violation or embracement of human dignity" was created. It was abstracted from the two descriptive themes: "Desiring to be seen as an equal human being" with the 
belonging categories of "Thoughts and assumptions on normality" and "Thoughts and assumptions on abnormality", and "Leading an overall good live in the chair" that was based and abstracted from the two following categories "Experiences of exclusion" and "Experiences of inclusion". This section elaborated these parts in more detail below. Please, see Table 1.

\subsection{Living in a Space between Potential Violation or Embracement of Human Dignity}

This theme of meaning was an interpretation of what the meaning of the participants' narrations in totally was. The finding showed that the informants were living a life where they constantly risked experiencing a violation of their birthright of human dignity or with the embracement of it.

\subsection{Desiring to Be Seen as an Equal and Worthy Human Being}

Cognitive preoccupation with what was normal versus abnormal, and related thoughts and assumptions on normality and on abnormality, was the dominant and prevailing aspect of the descriptive theme. It contained an underlining wish and desire of being seen as an equal and worthy human being regardless of the circumstance that they were electric wheelchair users. Freedom, respect, and their opposites were essential parts of the theme as were reflections on otherness or being different compared to others.

\subsubsection{Thoughts and Assumptions on Normality}

To be able to sense freedom and to feel free was an important part of life. It was described it as a part of the personality, and in that respect, it was essential to not be limited by the disability or the functioning variation. Moreover, it was important to be treated with respect and dignity. The category also included that participants did not want to live their lives "through" others. Another part of the normality was to be encountered and treated with respect by other people.

Table 1. Provides an overview of the sub-categories, categories, descriptive themes, and theme of meaning..

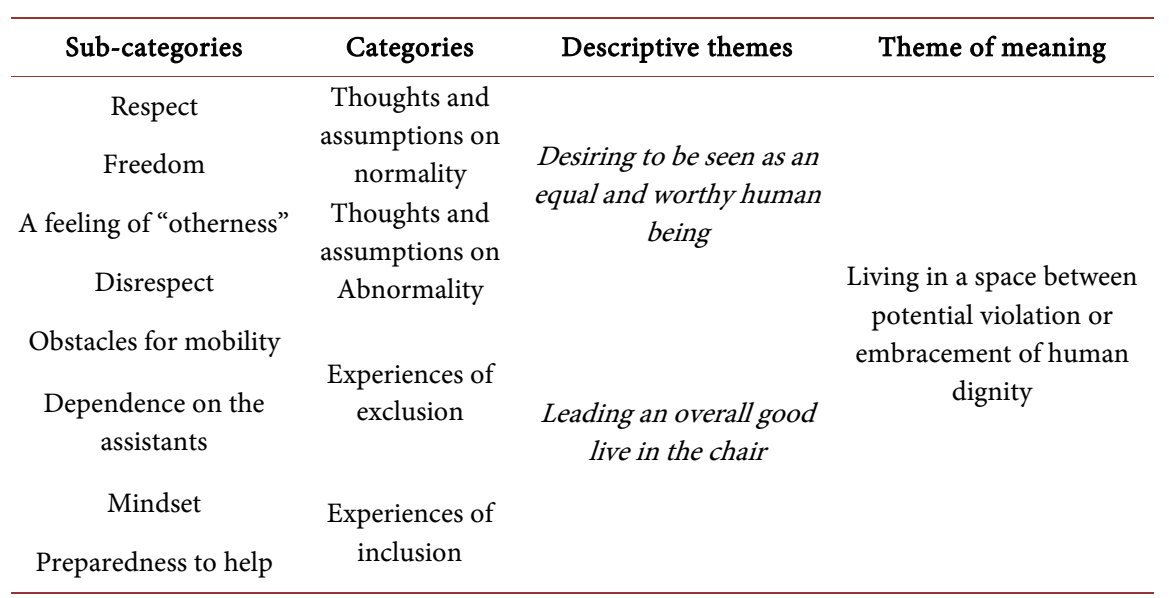


"I feel free in the electric chair" Another said: "Despite my handicap, I want to feel like a normal person and I do not want to live through another".

\subsubsection{Thoughts and Assumptions on Abnormality}

The findings showed that because of being in physically/spacial lower position as an electric wheelchair user, the informants sensed a feeling of "otherness", which made them highly sensitive to interpersonal interactions evolved. This, for instance, included that the end users wanted to be addressed directly, and not turning to the assistant with questions concerning the user by other people in the surrounding independently on the fact that they were situated in an electric wheelchair. If people addressed the assistant instead of the users personally, it confirmed the sense of "otherness" or "differentness". The sensitivity also included that they disliked if the assistants filled in sentences in their conversations with other people or if the assistants replied in their place. This meant that the surroundings did not await for the end user to answer. This was regarded a sign of disrespect and lack of understanding. The following two informant quotations, may serve as illusion of this category:

"I want to be me and I want them to talk to me" and "I do not want them to talk over my head".

One of the users perceived that there were still prejudices and strange attitudes to people who are wheelchair users.

"There are so many who think there's something wrong in the head just because you' re in a wheelchair'.

\subsection{Leading an Overall Good Live in the Wheel Chair}

In many ways, the informants recognized that they lead a good life. Nowadays, the participants described how their moved relatively freely. Sometimes, there could be difficulties to enter a restaurant as the door way was too small for the electric wheelchair or as there could be only one or two steps up or downwards. But, these were still hindrances that excluded the end users from full participation as the electric chairs are too heavy to carry.

\subsubsection{Experiences of Exclusion}

The positive and negative experiences co-existed and there where obstacles for mobility and dependence on assistants. The feeling of being outside or of being excluded because of the electric wheelchair came through. It could be too heavy to drive on gravel or on a soft lawn. One user told the following:

"We were at a party in a seabed and there was a stairway to a dock and then a big threshold, a manual chair could come up. However, I was excluded. The electric wheelchair was too big and heavy for lifting... Everywhere I sat outside and checked and the other was in there... then the electric chair was an obstacle".

The participants described being in a rather special position as they dependent on the assistants to different extends. Some of the users had assistants whom lived with them in their home almost 24 hours a day. One user experienced how 
the person felt particular vulnerable and dependent on other people. One of the reasons was also that the user had difficulty to raise the voice calling for help. This also led to a need of knowing where the assistants were physically located. One participant told about several incidences where the assistants several times had forgotten him/her. If the user and the assistant had been visiting a much-crowed place, for instance, a marked place, and the assistant saw a familiar person. The assistant could all of a sudden forget about his/her mission of working for the end user. This was experienced as peculiar. The assistants needed to know and to manage the balance between being supporting and not taking over. There were several examples from the users' perspective where the assistants had crossed the border of acceptable behaviors, for instance, when the user got a visitor, the assistant took over the conversation. Another aspect of the dependence of assistants dealt with neglect.

\subsubsection{Experiences of Inclusion}

Apart of this category dealt with how the assistants in one aspect were the prerequisite for the users to be included socially and societal, as were a good relationship between the end user and the assistants per se. This meant that the quality of the working relationship between the user and the assistant influenced the life and the quality of life for the users. If the relationships were good with the assistants, it was experienced as enriching instead. Further, the result showed that nowadays, it was experienced relatively accepted to be wheelchair-bound and quite easy to get help from the community, for example in connection with train or air travel. One of the participants explained.

"I can do what I want. I can fly to the heat and stay home and do what you want".

The participants also pointed at an experience of feeling safe, as people were generally nice and prepared to help. Another statement: "There is never any problem to sit in this chair. There are always people that can help me around".

Besides, the individuals' own mindset was outlined as an aspect of the experiences of inclusion-category. Here the approach the users took on to life was important. All informant described variations related the phenomenon of a process of acceptance with life circumstances.

"Well, there is no point in thinking, if I was "normal I could do this or that. I don' $t$ dwell about the fact that I am disabled, and that I cannot do this or that. I accept my destiny and I will make the best out of live".

\section{Discussion}

The study aimed to gain a deeper insight into electric wheelchairs users' perspectives and experiences of utilizing this device. The findings showed rather ambiguous and co-existing experiences of what it meant to be situated in an electric wheel chair as what were constantly important human values in play and on stake. The theme of meaning showed that the narrations expressed that it was like "Living in a space between potential violation and embracement of human 
dignity". This also mirrored the exposure to other peoples' interest, willingness and interaction skills. Further, the descriptive themes showed that the participants were desired to be seen as an equal and worthy human being, and at the same time they led an overall good live in the chair.

These experiences were interpreted as both respectful and freedom enhancing on the one hand, and as a sense of diversity and differentness as well as lack of respect on the other hand. The quality of the working relationship with between the users and the assistants immensely influenced the users' lives. A profound wish to be met with respect was seen. All are in line with the intent and ideology of the convention of people with disabilities [3]. It is also interesting to note that the findings showed an almost total absence of narrations about pain, and only to a little degree about physical problems.

\subsection{Basic Human Decency}

The narrations dealt with a mental preoccupation about what defines normality, and what was not regarded normal. Mostly, the relation to normality and abnormality as well as a fight of wanting to fit in was found. While, where was also a rest in the conviction that "I just want to be me", and that "I am satisfied with life". The findings articulated a wish to be regarded as normal/ordinary human beings, and it is not a surprising finding. According to Bronfenbrenner [22] all levels from micro to macro influence peoples' lives. The stories showed that the relation to the participants themselves were good at a micro level, but the ideation of how the macro level regarded the participants were more of a fight to "fit in", and as such being normal.

The importance of being able to decide for oneself and to be independent was seen here. It is close to the meaning, which Blach Rossen et al. [9] found as their participants also wanted autonomy and to function as independent individuals who had the opportunity to realize themselves.

\subsection{Accessibility}

The findings here showed that issues of inclusion and exclusion in term of being able to participate were part of the findings. This is further confirmed in Smith et al. [20] review that displayed a complexity of the body structures and functions, activity and participation, as well as and environmental and personal factors all influencing the inclusion and exclusion. Overall, the review showed that wheelchair factors and accessibility were the most frequently reported factors associated with participation. While, wheelchair skills, pain, finances and level of education were common modifiable factors. The authors pointed out the lack of efficacy studies in the relation to enhanced societal participation with interventions that should affect users' improved wheelchair confidence and/or lowered depression [20]. To enhance societal participation, service user involvement is need more as is currently not complicated enough [27].

Winance [28] stated that accessibility to places and services does not suffice to qualify as access for a particular person. A gap may emerge between the possibil- 
ities given by an environment and the real mobility of the person. The individual as an obstacle, a barrier, experiences this gap. In the social model of disability, this obstacle is objectified as being linked to the environment, with the solution being to eradicate disability to eliminate the obstacle, to modify the environment so that it is adapted to the individual's characteristics. The burden of adaptation is on the environment. The same argument is to be found in universal design, with all adaptive effort falling on the product: the product must be designed so that it is adapted to the diversity among users. This question is no longer "how to include diversity using the definition of universal", but "how to bring out and articulate this diversity, so as to vary the qualities of persons and the world in which they live". Implications for Rehabilitation "Inaccessibility" is analysed in terms of a discrepancy between the user and her/his environment and in terms of experience. Accessibility is seen as a process of adjustment and practical arrangements between the person and her/his environment. The universalism of Universal Design is criticized in the way it tries to force the variety of users into the uniqueness of one materiality. Emphasis is put on diversity and the necessity of articulating this diversity in a plural world offering various resources that individuals can call upon to act, depending on what they are and on what they want to become Winnance [28].

\section{Conclusion}

The study showed that electric wheelchair users were relatively content with their lives as well as with their devices in turns of mobility and accessibility, but the meaning of their narrations showed a life with at constant risk of having the respect of the human right and human respect violated. Besides, the importance of having access to good and high quality devices, good staffing, and environmental support, all in concordance with human rights, the clinical and practical implications of this study, narrows down to a question of encountering the other person as a whole and worthy individual.

\section{Future Research}

Future intervention-based research focusing on modifiable factors such as wheelchair skills and accessibility are warranted. Research studying the efficacy of improved confidence and/or lowered depression at enhancing participation is warranted. Experimental research focusing on modifiable factors is needed to further our understanding of factors influencing participation among wheelchair users, to enhance the knowledge of the social and community participation of wheelchair users [20].

\section{Methodological Considerations}

With regard to aspects of trustworthiness [1] [2] [23] [29], citations were presented for the reader to make independent judgement of the credibility. The promise not to reveal the identity of the three electric wheelchair users, led to a 
decision of not providing the reader with many details on the participants. Not describing the participants and their living context thoroughly can be seen as a weakness in qualitative content analysis, may lead to a problem for the reader to make a judgment of the study's credibility. Purposeful sampling was used for the identification and selection of information-rich cases for the most effective use of limited resources [29]. This involved identifying and selecting individuals or groups of individuals that are especially knowledgeable about or experienced with a phenomenon of interest and under study [23]. The participants confirmed the findings afterwards, and the content was presented and discussed with the research team. Further, the relatively rich and saturated findings may help the reader to decide on the transferability of the findings.

\section{Acknowledgements}

I will express my warmth gratitude to the participants, and their assistants whom made this first study possible. Further, I would like to thank the head of the municipal office of assistance, Mrs Linda Asplund, whom helped to identify possible participants for potential inclusion in this study. Further, I would like to thank the SMART research team, of which I am a part, for fruitful discussions about the meaning and contribution of this qualitative research.

His first study specifically oriented "Smart solutions for increased availability and quality for people with different kinds of disabilities requiring wheelchair based mobility SMART", which is related to a major research project SMART focusing on Internet of Things and SMART systems. The overarching purpose in the research project SMART is to increase awareness and knowledge of intelligent systems and services in the Mid Swedish region [30]. SMART systems include technologies such as measurement technology, embedded systems, wireless communications and the Internet of Things. Focus is on three distinct areas: Effective production (products for the energy efficiency/reduce emissions), Smart societies (products to reduce emission/resource use and increased accessibility for people with kind of disabilities), and Monitoring of large areas (products for energy efficiency and reduced impact on ecosystems). The general objective is to contribute to a sustainable society and increase the regions attractiveness and competitiveness through being national and international leading in smart systems for efficient production, smart societies and surveillance of large areas. Enhanced personal mobility, the ability to social interaction and to physical mobility are conjoint with active societal participation, which should include human beings regardless of any disability might be present.

\section{Author's Contribution}

LK designed and performed the collection of data, analysis and drafted the manuscript. The research team behind the author has been involved in discussions about recruitment of participants and preliminary results, though not consistently contributing to this paper. 


\section{References}

[1] Graneheim, U.H. and Lundman, B. (2004) Qualitative Content Analysis in Nursing Research: Concepts, Procedures and Measures to Achieve Trustworthiness. Nurse Education Today, 24, 105-112. https://doi.org/10.1016/j.nedt.2003.10.001

[2] Graneheim, U.H., Lindgren, B.-M. and Lundman, B. (2017) Methodological Challenges in Qualitative Content Analysis: A Discussion Paper. Nurse Education Today, 56, 29-34. https://doi.org/10.1016/j.nedt.2017.06.002

[3] United Nations (2006) Convention on the Rights of Persons with Disabilities. https://www.un.org/development/desa/disabilities/convention-on-the-rights-of-per sons-with-disabilities.html

[4] WHO (2001) International Classification of Functioning, Resolution WHA 54.21. Genèva.

[5] Peterson, D.B. (2005) International Classification of Functioning, Disability and Health: An Introduction for Rehabilitation Psychologists. Rehabilitation Psychology, 50, 105-112. https://doi.org/10.1037/0090-5550.50.2.105

[6] Schultz, I.Z., Stowell, A.W., Feuerstein, M. and Gatchel, R.J. (2007) Models of Return to Work for Musculoskeletal Disorders. Journal of Occupational Rehabilitation, 17, 327-352. https://doi.org/10.1007/s10926-007-9071-6

[7] Chan, F., Gelman, J.S., Ditchman, N., Kim, J.-H., Chiu, C.-Y., Chan, F., Da Silva Cardoso, E. and Chronister, J. A. (2009) Understanding Psychosocial Adjustment to Chronic illness and Disability. Springer, New York.

[8] Gudgeon, S. and Kirk, S. (2015) Living with a Powered Wheelchair: Exploring Children's and Young People's Experiences. Disability Rehabilitation: Assistive Technology, 10, 118-125.

http://www.permobil.com/Global/corpcom/pressrelease/Press\%20release_Dr.\%20G efen\%20receives\%20award.pdf https://doi.org/10.3109/17483107.2013.870609

[9] Blach Rossen, C., Sörensen, B., Jochumsen Wurst, B. and Wind, G. (2012) Everyday Life for Users of Electric Wheelchairs-A Qualitative Interview Study. Disability Rehabilitation: Assistive Technology, 7, 399-407 https://doi.org/10.3109/17483107.2012.665976

[10] Torkia, C., Reid, D., Korner-Bitensky, N., Kairy, D., Rushton, P.W., Demers, L. and Archambault, P.S. (2014) Power Wheelchair Driving Challenges in the Community: A Users' Perspective. Disability and Rehabilitation: Assistive Technology, 10, 211-215. https://doi.org/10.3109/17483107.2014.898159

[11] Stenberg, G., Henje, C., Levi, R. and Lindström, M. (2016) Living with an Electric Wheelchair-The User Perspective. Disability and Rehabilitation: Assistive Technology, 11, 385-394.

[12] Arbour-Nicitopoulos, K.P., Faulkner, G.E., Paglia-Boak, A. and Irving, H.M. (2010) Adolescents' Attitudes toward Wheelchair Users: A Provincial Survey. International Journal of Rehabilitation Research; 33, 261-263. https://doi.org/10.1097/MRR.0b013e328333de97

[13] Grindles, G.G., Wang, H., Jeannis, H., Teodorski, E. and Cooper, R.A. (2015) Design and User Evaluation of a Wheelchair Mounted Robotic Assisted Transfer Device. Biomedical Research International, 2015, Article ID: 198476. https://doi.org/10.1155/2015/198476

[14] Kirby, R.L. and Smith, C. (2001) Fall during a Wheelchair Transfer: A Case of Mismatched Brakes. The American Journal of Physical Medicine and Rehabilitation, 
80, 302-304. https://doi.org/10.1097/00002060-200104000-00015

[15] Ummat, S. and Kirby, R.L. (1994) Nonfatal Wheelchair-Related Accidents Reported to the National Electronic Injury Surveillance System. The American Journal of Physical Medicine and Rehabilitation, 73, 163-167. https://doi.org/10.1097/00002060-199406000-00004

[16] Xiang, H., Chany, A.-M. and Smith, G.A. (2006) Wheelchair Related Injuries Treated in US Emergency Departments. Injury Prevention, 12, 8-11. https://doi.org/10.1136/ip.2005.010033

[17] Nicholson, N.R. (2009) Social Isolation in Older Adults: An Evolutionary Concept Analysis. Journal of Advanced Nursing, 65, 1342-1352. https://doi.org/10.1111/j.1365-2648.2008.04959.x

[18] Commission of the European Communities (2000) Social Policy Agenda: Communication from the Commission to the Council, the European Parliament, the Economic and Social Committee and the Committee of the Regions. Commission of the European Communities, Brussels.

[19] Ripat, J.D., Brown, C.L. and Ethans, K.D. (2015) Barriers to Wheelchair Use in the Winter. Archives of Physical Medicine \& Rehabilitation, 96, 1117-1122. https://doi.org/10.1016/j.apmr.2015.01.020

[20] Smith, E.M., Sakakibara, B.M. and Miller, W.C. (2016) A Review of Factors Influencing Participation in Social and Community Activities for Wheelchair Users. Disability and Rehabilitation Assistive Technology, 11, 361-374.

[21] Stokols, D. (1996) Translating Socialecological Theory into Guidelines for Community Health Promotion. American Journal of Health Promotion, 10, 282-298. https://doi.org/10.4278/0890-1171-10.4.282

[22] Bronfenbrenner, U. (1979) The Ecology of Human Development: Experiments by Nature and Design. Harvard University Press, Cambridge.

[23] Creswell, J.W. and Poth, J. (2018) Qualitative Inquiry \& Research Design-Choosing among Five Approaches. 4nd Edition, Sage Publications, London.

[24] Polit, D.F. and Beck, C.T. (2015) Nursing Research. Principles and Methods. 15th Edition, J.B. Lippincott Company, Philadelphia.

[25] Krippendorff, K. (2013) Content Analysis: An Introduction to Its Methodology. Third Edition, SAGE Publications, Thousand Oaks.

[26] (2016) The Madrid Declaration on Ethical Standards for Psychiatric Practice. http://www.wpanet.org/detail.php?section_id=5\&content_id=48

[27] Bee, P., Price, O., Baker, J. and Lovell, K. (2015) Systematic Synthesis of Barriers and Facilitators to Service User-Led Care Planning. British Journal Psychiatry, 207, 104-114. https://doi.org/10.1192/bjp.bp.114.152447

[28] Winance, M. (2014) Universal Design and the Challenge of Diversity: Reflections on the Principles of UD, Based on Empirical Research of People's Mobility. Disability \& Rehabilitation, 36, 1334-1343. https://doi.org/10.3109/09638288.2014.936564

[29] Patton, M.Q. (2015) Qualitative Research \& Evaluation Methods. 4th Edition, Sage Publications, London.

[30] Mid Sweden University (2016). https://www.miun.se/en/stc-research-centre/Research-Projects/smart/ 